\title{
Identification of two novel brominated contaminants in water samples by ultra-high performance liquid chromatography-Orbitrap Fusion Tribrid mass spectrometer
}

\author{
Aifeng Liu, Guangbo Qu, Chaoli Zhang, Yan Gao, Jianbo Shi*, Yuguo Du, Guibin Jiang \\ State Key Laboratory of Environmental Chemistry and Ecotoxicology, Research Center for Eco-Environmental Sciences, Chinese Academy of Sciences, \\ P.O. Box 2871, Beijing 100085, China
}

\section{A R T I C L E I N F O}

\section{Article history:}

Received 17 September 2014

Received in revised form

26 November 2014

Accepted 10 December 2014

Available online 18 December 2014

\section{Keywords:}

Orbitrap Fusion Tribrid mass spectrometer Impurities

Tetrabromobisphenol A derivatives

Water samples

\begin{abstract}
A B S T R A C T
Tetrabromobisphenol A mono(2-hydroxyethyl ether) (TBBPA-MHEE) and TBBPA mono(glycidyl ether) (TBBPA-MGE), two impurities of TBBPA derivatives, were hypothesized to be novel brominated contaminants with potential toxicity. However, due to lacking of analytical method and pure standards, their environmental behavior and toxicity have not been studied. Herein we developed a sensitive method based on ultra-high performance liquid chromatography-Orbitrap Fusion Tribrid mass spectrometer (UHPLC-Orbitrap Fusion TMS) for simultaneous detection of TBBPA-MHEE and TBBPA-MGE in water samples. The sample pretreatment method and the experimental conditions of UHPLC and Orbitrap Fusion TMS, were optimized in detail. The instrument detection limits (IDLs) for TBBPA-MHEE and TBBPA-MGE were $0.5 \mathrm{pg}$ and $0.6 \mathrm{pg}$, respectively. The method detection limits (MDLs) for TBBPA-MHEE and TBBPA-MGE in river water samples were 0.9 and $0.8 \mathrm{ng} / \mathrm{L}$. With the proposed method, we were able to detect TBBPA-MHEE and TBBPA-MGE for the first time in water samples and technical products of TBBPA derivatives. Therefore, UHPLC-Orbitrap Fusion TMS is a simple and effective tool for identification and quantification of novel contaminants in the environment.
\end{abstract}

(c) 2014 Elsevier B.V. All rights reserved.

\section{Introduction}

As one of the most important brominated flame retardants (BFRs), tetrabromobisphenol A (TBBPA) has caused widespread concern for its extensive use and potentially environmental and health risks [1,2]. Approximately $18 \%$ of TBBPA has been applied to produce derivatives, such as brominated epoxy resins, TBBPA bis(2-hydroxyethyl ether) (TBBPA-BHEE), TBBPA bis(allyl ether) (TBBPA-BAE) and TBBPA bis(2,3-dibromopropyl ether) (TBBPABDBPE) [1,2], which have been found in various matrices [3-8].

With the BFRs releasing into the environment, many impurities associated with their synthesis are becoming a concern as well for their potential exposure to humans and wildlife [9-11]. In our previous work, we identified TBBPA mono(allyl ether) (TBBPAMAE) and TBBPA mono(2,3-dibromopropyl ether) (TBBPA-MDBPE) as impurities of TBBPA-BAE and TBBPA-BDBPE, with the ability of accumulation in the biota system [12]. Most of the derivatives are produced using TBBPA as the raw material. The impurities,

\footnotetext{
* Corresponding author. Tel.: +86 10 62849129; fax: +861062849129.

E-mail address: jbshi@rcees.ac.cn (J. Shi).
}

generally mono-modified TBBPA analogs, would be coproduced with the derivatives when the reaction of TBBPA with the corresponding reagents is incomplete. Because of the similar production process of TBBPA derivatives, we hypothesized that TBBPA mono(2hydroxyethyl ether) (TBBPA-MHEE) and TBBPA mono(glycidyl ether) (TBBPA-MGE) were two impurities of TBBPA-BHEE and TBBPA bis(glycidyl ether) (TBBPA-BGE), which are widely used to produce engineering polymers, epoxy resins, etc. $[1,2,13]$ Because of the similarity of the structures, TBBPA-MHEE and TBBPA-MGE have the similar physical-chemical properties with TBBPA and derivatives and potential toxicity. In order to further study their environmental sources, behavior and toxicity, a sensitive analytical method is urgently needed.

TBBPA and its derivatives are often determined with highperformance liquid chromatography coupled with tandem mass spectrometry (HPLC-MS/MS) and gas chromatography coupled with mass spectrometry (GC-MS) $[5,14,15]$. For the detection of TBBPA with GC-MS, the derivatization process is essential which limited the applicability of the method [2]. In addition, selective reactive monitoring (SRM) or multi-reaction monitoring (MRM) mode is usually used for the quantification of BFRs [16-18]. However, the quantification of trace level target compounds in full scan 
mass mode is impossible because of the relatively low resolution and high noise. Therefore, only the selected parent ions are allowed to transform to the unique fragment ions in the third quadrupole. As a result, a large amount of information is missed because only specific ion transition processes are monitored [19]. Moreover, the optimization of the parameters for each target compounds in tandem mass spectrometry is a time-consuming process.

In recent years, Orbitrap high resolution mass spectrometry (HRMS) has been rapidly developed to perform analysis in full scan acquisition mode. Orbitrap HRMS could provide higher resolution ( $\geq 50,000 \mathrm{FWHM}$ at $\mathrm{m} / \mathrm{z} 200$ ) than time of flight (TOF) HRMS and give qualitative information with comparable analysis accuracy, selectivity and sensitivity as with HPLC-MS/MS [19-23]. For example, the Orbitrap Fusion Tribrid mass spectrometer (Orbitrap Fusion TMS) can provide even higher specificity and sensitivity with a reliable high resolution up to 450,000 FWHM at m/z 200 . Although Orbitrap HRMS was mainly used for the analysis of drugs and metabolites [19,24,25], it was also reported as appropriate for identification and quantification of environment contaminants $[26,27]$. For the analysis of small molecules, the scan rate of Orbitrap Fusion TMS is recommended to be set at normal with a resolution of 120,000 FWHM which could guarantee both the scan rate and sensitivity.

In this study, we first synthesized TBBPA-MHEE and TBBPAMGE, and then we developed a novel method for simultaneous detection of the above two compounds with ultra-high performance liquid chromatography-Orbitrap Fusion TMS (UHPLCOrbitrap Fusion TMS). The experimental conditions were optimized in detail. With the proposed method, we were able to identify TBBPA-MHEE and TBBPA-MGE in water samples and technical TBBPA derivatives. To our knowledge, this is the first report of an analytical method for TBBPA-MHEE and TBBPA-MGE detection, and their relative mass spectra, along with their occurrence in the environmental matrices. This study also demonstrates that UHPLCOrbitrap TMS is a powerful tool for the identification of novel brominated contaminants related to TBBPA derivatives.

\section{Experimental}

\subsection{Chemicals and materials}

TBBPA (98\%) was purchased from Alfa Aesar and used without further purification. $\mathrm{NaCl}(\geq 99.5 \%), \mathrm{HCl}(37 \%)$ and ammonium hydroxide $(50 \% \mathrm{~V} / \mathrm{V}$, water solution) were purchased from Sigma-Aldrich. All standards were dissolved in methanol at a mass concentration of $10 \mathrm{mg} / \mathrm{mL}$ and stored in a refrigerator at $4{ }^{\circ} \mathrm{C}$. All calibration standards and spiking solutions were prepared by serial dilution in methanol. Methanol, acetone and dichloromethane (DCM) were all HPLC grade. Water was generated by a Milli-Q advantage $\mathrm{A} 10$ system.

\subsection{The synthesis procedures of TBBPA-MHEE and TBBPA-MGE}

TBBPA-MHEE and TBBPA-MGE were synthesized in our laboratory and further characterized by ${ }^{1} \mathrm{H}$ nuclear magnetic resonance ( ${ }^{1}$ HNMR) and UHPLC-Orbitrap Fusion TMS (Ultimate 3000 UHPLC, Orbitrap Fusion Tribrid mass spectrometer, Thermo Fisher Scientific, USA). The purities of two synthesized compounds, determined by HPLC (UV $214 \mathrm{~nm}$, Thermo Ultimate 3000 HPLC, Thermo Fisher Scientific, USA), were $98 \%$ and $99 \%$, respectively. TBBPA-MHEE and TBBPA-MGE were synthesized following the schemes in Fig. S1 in Supporting Information.

\subsubsection{TBBPA-MHEE}

Sodium hydroxide solution (168 $\mathrm{mg}$ in $1.2 \mathrm{~mL}$ water) was added at room temperature to a solution of TBBPA $(1.09 \mathrm{~g})$ and 2-bromoethanol $(160 \mu \mathrm{L})$ in acetone $(5 \mathrm{~mL})$. The reaction mixture was stirred for $5 \mathrm{~h}$. After the TLC showed the disappearance of TBBPA, the reaction mixture was diluted with DCM and washed with saturated solution of sodium bicarbonate and water. The organic layer was dried over anhydrous $\mathrm{MgSO}_{4}$. After removal of the organic solvent, the residue was purified by column chromatography, giving TBBPA-MHEE at a yield of $48 \%$.

\subsubsection{TBBPA-MGE}

M-CPBA (m-Chloroperbenzoic acid, 70\%, $259 \mathrm{mg}$ ) dissolved in $5 \mathrm{~mL}$ DCM was added dropwise to a TBBPA-MAE ( $584 \mathrm{mg})$ solution in $5 \mathrm{~mL} \mathrm{DCM}$ and the mixture was stirred at room temperature for $5 \mathrm{~h}$. After the TLC showed the disappearance of the reagent, the reaction mixture was diluted with $30 \mathrm{~mL}$ DCM and washed with saturated sodium thiosulfate, saturated sodium bicarbonate and water. The organic layer was dried over anhydrous $\mathrm{MgSO}_{4}$. After removal of the organic solvent, the residue was purified by column chromatography, giving TBBPA-MGE at a yield of 50\%.

\subsection{Instrumental analysis}

\subsubsection{Instrumental parameters of UHPLC-Orbitrap Fusion TMS}

We employed a Thermo Ultimate 3000 UHPLC coupled with a Orbitrap Fusion TMS (Orbitrap Fusion Tribrid mass spectrometer, Thermo Fisher Scientific, USA) and ZORBAX ODS column $(150 \times 3 \mathrm{~mm}, 5 \mu \mathrm{m}$, Agilent) for the analysis of the environmental samples and technical products. Methanol (A) and water (B) were applied as mobile phases at a flow rate of $0.6 \mathrm{~mL} / \mathrm{min}$. The mobile phase gradient was initially $80: 20(\mathrm{v} / \mathrm{v}, \mathrm{A} / \mathrm{B})$ and kept for $2 \mathrm{~min}$, then increased to $100 \% \mathrm{~A}$ along $5 \mathrm{~min}$ and returned to $80: 20(\mathrm{v} / \mathrm{v}, \mathrm{A} / \mathrm{B})$ in $1 \mathrm{~min}$ and then kept for 2 additional min. Electrospray ionization was used as the ionization method and operated in the negative ion mode. The recommended values were used in the full scan acquisition without further optimization. TBBPA-MHEE and TBBPA-MGE were analyzed at full-scan mode from m/z 100 to 1000 under heated electrospray ionization (HESI) mode. Orbitrap Fusion TMS had two fragmentation techniques, collision induced dissociation (CID) and higher-energy collisional dissociation (HCD). Little information about the daughter ions was observed when CID was used, thus HCD was used as the fragmentation technique. The parameters used for the mass spectrometer were the following: spray voltage, $2500 \mathrm{~V}$; sweep gas flow rate, 1 respective arbitrary units; sheath gas flow rate, 40 respective arbitrary units; aux gas flow rate, 10 respective arbitrary units; ion transfer tube temperature, $350^{\circ} \mathrm{C}$; vaporizer temperature, $200^{\circ} \mathrm{C}$; $\mathrm{MS}^{1}$ detector, Orbitrap; $\mathrm{MS}^{1}$ resolution, 120,000; $\mathrm{MS}^{1}$ scan range, 100-1000; $\mathrm{MS}^{1}$ maximum injection time, $100 \mathrm{~ms}$; $\mathrm{MS}^{1}$ automated gain control (AGC) target, 100,000; S-lens RF level, $60 \mathrm{~V}$; $\mathrm{MS}^{2} \mathrm{HCD}$ collision energy, 45\%; $\mathrm{MS}^{2}$ detector, Orbitrap; $\mathrm{MS}^{2}$ resolution, 15,000; $\mathrm{MS}^{2}$ AGC target, 50,000; $\mathrm{MS}^{2}$ maximum injection time, $35 \mathrm{~ms}$; $\mathrm{MS}^{2}$ start mass, 50 . Xcalibur Qual and Quan Browser software were used for the qualitative and quantitative analysis.

\subsubsection{The instrument condition, elution program and the optimized parameters employed in HPLC-MS/MS analysis}

A Waters 2695 HPLC coupled with a triple-quadrupole mass spectrometer (Quattro Premier XE, Waters, USA) was used to compare the instrument detection limits (IDLs) with that of the UHPLC-Orbitrap Fusion TMS. The same chromatographic column and mobile phase gradient were applied for the MS/MS analysis. MS/MS parameters were optimized in infusion experiments using an individual standard solution at a mass concentration of $100 \mathrm{ng} / \mathrm{mL}$. The precursor ion was obtained from the mass scan mode with optimized parameters for cone voltage (optimized from $-10 \mathrm{~V}$ to $-60 \mathrm{~V}$ ) and capillary voltage (optimized from $1.0 \mathrm{kV}$ to $5.0 \mathrm{kV}$ ). The Mass scan mode was run by scanning from $\mathrm{m} / \mathrm{z} 50$ to 
1000 , using a scan time of $0.2 \mathrm{~s}$ with a step size of $0.1 \mathrm{amu}$ and a pause of $100 \mathrm{~ms}$ between two scans. Fragment ion was optimized by the collision energy to obtain the best ion intensity in daughter scan mode. The capillary voltage was $2.5 \mathrm{kV}$, source temperature and desolvation temperature were $120^{\circ} \mathrm{C}$ and $450^{\circ} \mathrm{C}$, respectively. Desolvation gas and cone gas flow were $400 \mathrm{~L} / \mathrm{h}$ and $100 \mathrm{~L} / \mathrm{h}$, respectively. Argon gas, used for the ionization of daughter ion in the collision cell, was set at a flow rate of $0.25 \mathrm{~L} / \mathrm{h}$. The MRM mode was used for the quantification of target compounds. For TBBPAMHEE, the optimized cone voltage was $-42 \mathrm{~V}$ for the parent ion (586.7), the optimized collision energy for quantitative ion (526.4) and qualitative ion (541.6) were -45 and $-30 \mathrm{eV}$, respectively. For TBBPA-MGE, the optimized cone voltage was $-38 \mathrm{~V}$ for the parent ion (598.7), the optimized collision energy for quantitative ion (526.4) and qualitative ion (541.6) were -45 and $-28 \mathrm{eV}$, respectively.

\subsection{Sample collection}

Three BFRs factories and three rivers near them in Shouguang, Shandong Province were selected to collect waste water and river water samples. Four sea water samples were collected in Yantai, Shandong Province.

Five technical products including TBBPA-BHEE and brominated epoxy resins were also purchased to determine the mass concentration of impurities.

\subsection{Sample pretreatment}

\subsubsection{Solid phase extraction (SPE) procedures}

The $\mathrm{pH}$ values of the water samples were adjusted to 4-5 with $1 \mathrm{M} \mathrm{HCl}$ after they were filtered through a $0.45 \mu \mathrm{m}$ glass fiber membrane filter. Then, Water samples $(200 \mathrm{~mL}$ each $)$ were treated with SPE cartridges (HLB cartridges, Waters Oasis, $500 \mathrm{mg}$, $6 c c$; 18 cartridges, Waters Sep-Pak Vac 6cc, $500 \mathrm{mg}$; ENVI ${ }^{\mathrm{TM}}$ carb SPE tubes, Supelclean ${ }^{\mathrm{TM}}, 0.5 \mathrm{~g}, 6 \mathrm{~mL}$ ). The cartridges were first conditioned with $10 \mathrm{~mL}$ methanol, and then $10 \mathrm{~mL}$ Milli-Q water. The water samples were loaded on the cartridges under vacuum condition at a flow rate of about $5 \mathrm{~mL} / \mathrm{min}$. After sampling, cartridges were washed with $10 \mathrm{~mL} 5 \%$ methanol/water to eliminate interferences. The cartridges were then vacuumed for $30 \mathrm{~min}$ to remove most of the water and eluted with $10 \mathrm{~mL}$ elution solvent. For the optimization of elution solvents, methanol, $0.5 \%-10 \%$ ammonium hydroxide/methanol, acetone and 0.5\% ammonium hydroxide/acetone were used. Prior to analysis by UHPLC-Orbitrap Fusion TMS, the eluates were concentrated to $1 \mathrm{~mL}$ under a gentle nitrogen gas flow.

\subsubsection{Liquid-liquid extraction}

Water samples $(200 \mathrm{~mL})$ were first filtered through a $0.45 \mu \mathrm{m}$ glass fiber membrane filter. Then, the $\mathrm{pH}$ was adjusted to 4-5 with $1 \mathrm{M} \mathrm{HCl}$, and $20 \mathrm{~g} \mathrm{NaCl}$ were added. The samples were then extracted three times with $100 \mathrm{~mL}$ DCM. The organic phases were combined, reduced to approximately $1 \mathrm{~mL}$ with a rotary evaporator and blown dried with a gentle nitrogen flow. The solvent was changed to $1 \mathrm{~mL}$ methanol prior to the analysis by UHPLC-Orbitrap Fusion TMS.

\section{Results and discussion}

\subsection{Characterization of TBBPA-MHEE and TBBPA-MGE}

TBBPA-MHEE and TBBPA-MGE were characterized by ${ }^{1} \mathrm{HNMR}$ and Orbitrap Fusion TMS. The detailed data are described in Supporting Information.
${ }^{1} \mathrm{HNMR}$ results showed successful synthesis and high purity of both TBBPA-MHEE and TBBPA-MGE (Fig. S1). The HPLC (UV $214 \mathrm{~nm}$ ) determination further confirmed the purities of TBBPA-MHEE and TBBPA-MGE were 98\% and 99\%, respectively. UHPLC-Orbitrap Fusion TMS spectra (Fig. 1) further confirmed the identities of these two compounds. The phenolic hydroxyl groups of TBBPAMHEE and TBBPA-MGE were easily ionized and formed the ion clusters $[\mathrm{M}-\mathrm{H}]^{-}$which were similar to those of TBBPA, TBBPAMAE and TBBPA-MDBPE under ESI mode $[12,28]$. The ions at $\mathrm{m} / \mathrm{z}$ 586.77185 for TBBPA-MHEE and 598.77191 for TBBPA-MGE in the full scan mass spectra (m/z 100-1000) could be explained by $[\mathrm{M}-\mathrm{H}]^{-}$. The results showed that the $\mathrm{m} / \mathrm{z}$ values were the most consistent with the calculated ones which were 586.77192 and 598.77192 for TBBA-MHEE and TBBPA-MGE, respectively. The isotopic $\mathrm{Br}$ distribution (1:4:6:4:1) demonstrated the presence of four bromine atoms in TBBPA-MHEE and TBBPA-MGE. The daughter ion clusters of 586.77 and 598.77 are shown in Fig. 1. TBBPA-MHEE and TBBPA-MGE had similar daughter ion clusters at $\mathrm{m} / \mathrm{z} 541.74,526.71,448.80,290.89 / 290.88$ and $78.92 / 80.92$, which indicated cleavage of the ether bond, breakage of the methyl, and cleavage of the bond between carbon and bromine ion. The molecular formulas of the daughter ions were calculated with Xcalibur Qual Browser software based on the accurate $\mathrm{m} / \mathrm{z}$ value UHPLC-Orbitrap Fusion TMS detected. The possible structures of the daughter ions are given in Fig. 1 according to their molecular formulas and the similar fragmentation properties which previous reported for TBBPA [29]. Therefore, TBBPA-MHEE and TBBPA-MGE were used as the standards throughout the experiments because of the high-quality synthesis of these two chemicals.

\subsection{Optimization of sample extraction and cleanup}

For extraction of water samples, solid phase extraction (waters HLB cartridges, Waters Oasis, $500 \mathrm{mg}$, 6cc; C18 cartridges, Waters Sep-Pak Vac 6cc, 500 mg; ENVI ${ }^{\mathrm{TM}}$-carb SPE tubes, Supelclean ${ }^{\mathrm{TM}}$, $0.5 \mathrm{~g}, 6 \mathrm{~mL}$ ) and liquid-liquid extraction were compared. TBBPAMHEE and TBBPA-MGE were spiked in river water samples at a mass concentration of $50 \mathrm{ng} / \mathrm{L}$. The recovery data about the SPE are shown in Table S1 in Supporting Information. For the optimization of elution solvent, methanol, $0.5 \%, 1 \%, 5 \%$ and $10 \%$ ammonium hydroxide/methanol, acetone and $0.5 \%$ ammonium hydroxide/acetone were used. HLB cartridges showed mean recoveries ranging from $15 \%$ to $40 \%$ for TBBPA-MHEE and from $12 \%$ to $18 \%$ for TBBPA-MGE. C18 cartridges showed mean recovery ranging from $16 \%$ to $41 \%$ for TBBPA-MHEE and from $15 \%$ to $31 \%$ for TBBPAMGE. ENVI ${ }^{\mathrm{TM}}$-carb SPE tubes showed recovery lower than $14 \%$ for TBBPA-MHEE and lower than $8 \%$ for TBBPA-MGE. With the use of ammonium hydroxide, no obvious improvement of the recoveries was observed. However, in order to blow off $10 \mathrm{~mL}$ methanol contained $10 \%$ ammonium hydroxide, time longer than $2 \mathrm{~h}$ and much nitrogen gas were imperative. If the ammonium hydroxide did not volatilize completely, it would affect the retention time of target compounds in UHPLC analysis. In addition, the absorbent efficiency of cartridges was also determined. TBBPA-MHEE and TBBPA-MGE were spiked in $10 \mathrm{~mL}$ deionized water at a mass concentration of $10 \mu \mathrm{g} / \mathrm{L}$ and loaded onto the three kinds of SPE cartridges. We collected the eluted water and the eluted $5 \%$ methanol/water section, and analyzed with UHPLC-Orbitrap Fusion TMS. TBBPA-MHEE and TBBPA-MGE could not be detected at these two elution composition. The results indicated TBBPA-MHEE and TBBPA-MGE strongly absorbed to the sorbents of the cartridges and were difficult to be eluted. Therefore, SPE might not be an appropriate extraction method for the trace level detection of TBBPA-MHEE and TBBPAMGE in water samples. 
34-200PPB \#1957 RT: 3.53 AV: 1 NL: 4.14E6

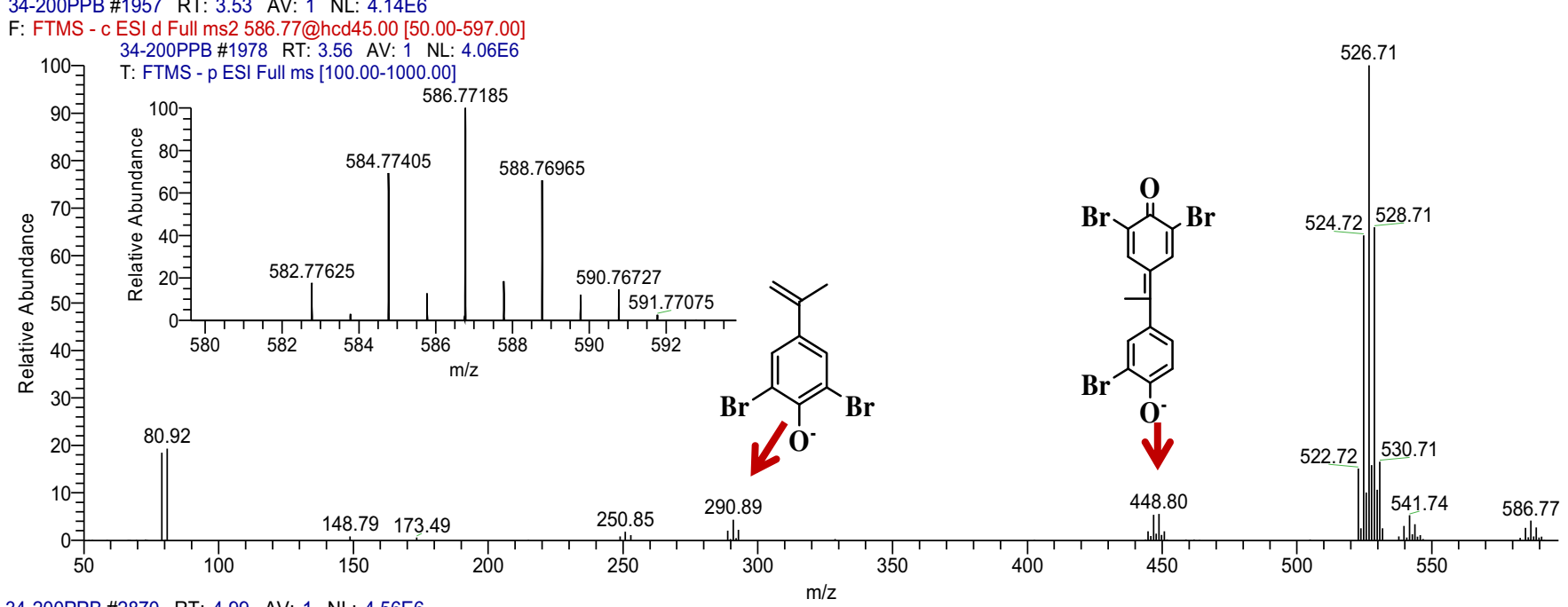

34-200PPB \#2870 RT: 4.99 AV: 1 NL: 4.56E6

F: FTMS - c ESI d Full ms2 598.77@hcd45.00 [50.00-609.00]

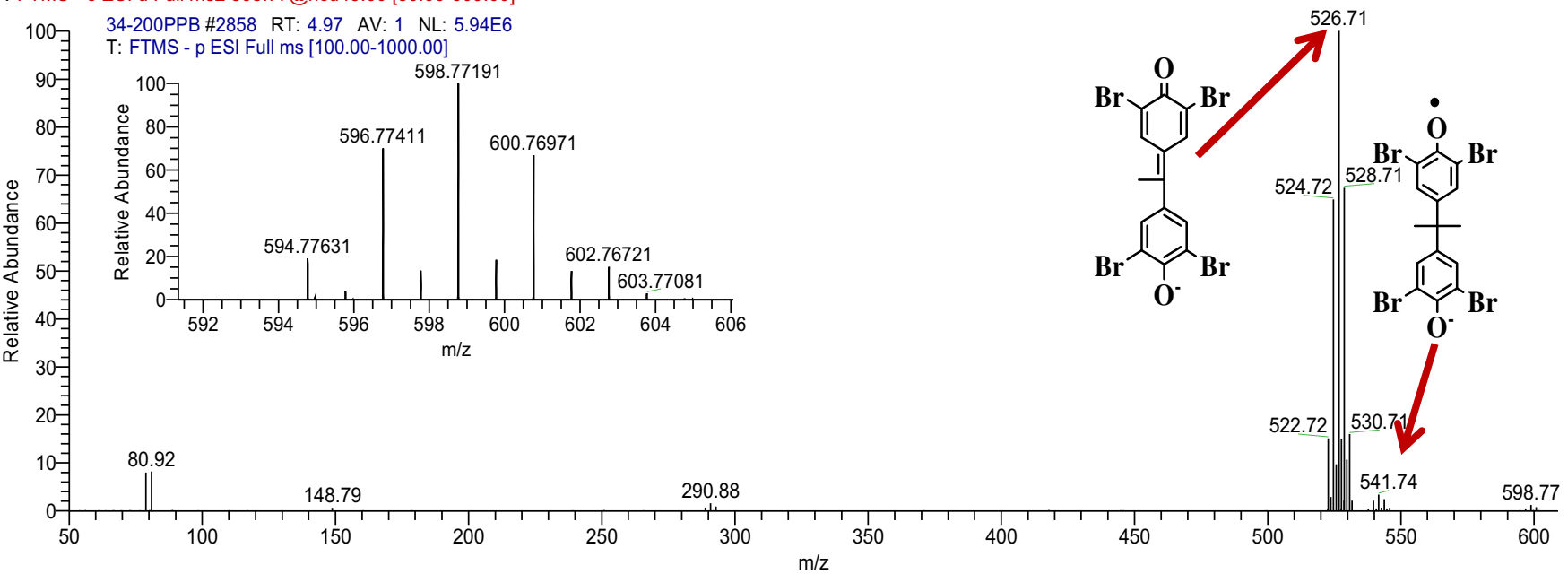

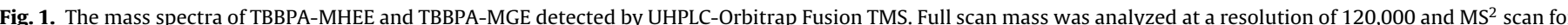
parent ions were detected at a resolution of 15,000

Therefore, liquid-liquid extraction was selected with DCM as the extraction solvent for the extraction of waste water. Salt addition is a common method for the improvement of extraction efficiency because $\mathrm{NaCl}$ could reduce the solubility of organic molecules in aqueous system and improve their distribution in organic phase $[30,31]$. Ten percent of $\mathrm{NaCl}$ was dissolved in water samples to assist the extraction process. Although an improvement in the recovery was not observed, it was useful for the layer

Table 1

The recoveries and matrix effects of TBBPA-MHEE and TBBPA-MGE.

\begin{tabular}{|c|c|c|c|}
\hline Sample Type & & TBBPA-MHEE & TBBPA-MGE \\
\hline \multirow{6}{*}{$\begin{array}{l}\text { Waste water } \\
\left(\mathrm{MR}^{\mathrm{a}} \pm \mathrm{SD}\right)\end{array}$} & Calibration curves & $y=(3.94 \mathrm{E}-06 \pm 6.76 \mathrm{E}-08) x-(0.093 \pm 1.191)$ & $y=(4.78 \mathrm{E}-06 \pm 1.10 \mathrm{E}-07) x-(0.227 \pm 1.679)$ \\
\hline & $R^{2}$ & 0.9940 & 0.9958 \\
\hline & Linearity range & $0.2-200 \mathrm{ng} / \mathrm{mL}$ & $0.2-200 \mathrm{ng} / \mathrm{mL}$ \\
\hline & $500 \mathrm{ng} / \mathrm{L}, n=5$ & $75 \pm 5 \%$ & $76 \pm 13 \%$ \\
\hline & $50 \mathrm{ng} / \mathrm{L}, n=7$ & $79 \pm 8 \%$ & $84 \pm 12 \%$ \\
\hline & $5 \mathrm{ng} / \mathrm{L}, n=7$ & $59 \pm 5 \%$ & $101 \pm 14 \%$ \\
\hline \multirow{4}{*}{$\begin{array}{l}\text { Tap water } \\
(\mathrm{MR} \pm \mathrm{SD}) \\
\text { Waste water } \\
\left(\mathrm{ME}^{\mathrm{b}} \pm \mathrm{SD}\right)\end{array}$} & $500 \mathrm{ng} / \mathrm{L}, n=9$ & $85 \pm 4 \%$ & $85 \pm 6 \%$ \\
\hline & $0.2 \mathrm{ng} / \mathrm{mL}, n=3$ & $0.93 \pm 0.05$ & $1.03 \pm 0.04$ \\
\hline & $1 \mathrm{ng} / \mathrm{mL}, n=3$ & $0.95 \pm 0.06$ & $0.97 \pm 0.04$ \\
\hline & $20 \mathrm{ng} / \mathrm{mL}, n=3$ & $1.01 \pm 0.03$ & $1.08 \pm 0.03$ \\
\hline \multirow{3}{*}{$\begin{array}{l}\text { River water } \\
(\mathrm{ME} \pm \mathrm{SD})\end{array}$} & $0.2 \mathrm{ng} / \mathrm{mL}, n=3$ & $0.95 \pm 0.04$ & $0.96 \pm 0.05$ \\
\hline & $1 \mathrm{ng} / \mathrm{mL}, n=3$ & $0.91 \pm 0.08$ & $1.10 \pm 0.04$ \\
\hline & $20 \mathrm{ng} / \mathrm{mL}, n=3$ & $0.98 \pm 0.03$ & $1.03 \pm 0.02$ \\
\hline
\end{tabular}

a Mean recovery (200 $\mathrm{mL}$ water sample).

b Matrix effect. 
separation of the DCM and the water samples. The partition of phenol in aqueous solution could be positively affected by $\mathrm{pH}$ value, and phenols are inclined to participate in organic phase when they are extracted from acidic solution [32,33]. Herein, before the extraction procedure, $\mathrm{HCl}$ solution $(1 \mathrm{M})$ was used to adjust the $\mathrm{pH}$ of the water samples to 4-5. The recoveries of TBBPA-MHEE and TBBPA-MGE (Table 1) were $75 \%$ and $76 \%$ at the mass concentration of $500 \mathrm{ng} / \mathrm{L}$, $79 \%$ and $84 \%$ at the mass concentration of $50 \mathrm{ng} / \mathrm{L}$, and $59 \%$ and $101 \%$ at the mass concentration of $5 \mathrm{ng} / \mathrm{L}$, respectively.

\subsection{Quality control and method performance}

Least squared regression analysis showed good determination coefficient $\left(R^{2}\right)$ higher than 0.99 (Table 1$)$. An external standard method and a linear calibration curve with ten points for TBBPAMHEE and TBBPA-MGE (Table 1 , from 0.2 to $200 \mathrm{ng} / \mathrm{mL}, 5 \mu \mathrm{L}$ injected) were used to quantify the target compounds. In order to guarantee no cross contamination, groups of 5 samples were prepared with one blank river water sample, and analyzed with methanol as solvent blank.

The quantification of the target compounds was based on the high resolution of UHPLC-Orbitrap Fusion TMS which was operated at a resolution of $120,000 \mathrm{FWHM}$. The $\mathrm{m} / \mathrm{z}$ was extracted from the total ion chromatography with a mass tolerance of $3 \mathrm{ppm}$. UHPLC-Orbitrap Fusion TMS could positively overcome the disadvantage of the low resolution mass spectrometer when the quantification was operated in mass scan mode [19]. The target compounds could be effectively distinguished from the interfering substances with the closest $\mathrm{m} / \mathrm{z}$ value. TBBPA-MHEE and TBBPA-MGE in samples were identified by retention time, isotope distribution, accurate $\mathrm{m} / \mathrm{z}$ of the parent ions and relative daughter ions comparison with the corresponding standards. Quantification of the target compounds in the environmental samples was performed by peak area of the accurate parent ions with $3 \mathrm{ppm}$ mass tolerance, and 586.77185 and 598.77191 were used as quantitative ions for TBBPA-MHEE and TBBPA-MGE. The isotope peaks at $\mathrm{m} / \mathrm{z} 584.77405$ and 596.77411 were used for the qualitative ions for TBBPA-MHEE and TBBPA-MGE, respectively. The bromine isotope distributions of TBBPA-MHEE and TBBPA-MGE were also used as an important property for their identification in environment samples. Because of the high resolution of the instrument, sometimes no noise exists especially when the mass concentration of the analyte is very low. The detection limits (DLs) were determined by the lowest mass value of the target compounds that Orbitrap Fusion TMS detected. The IDLs were detected for 5 times within the $20 \%$ relative standard deviation for the signals. The IDLs were $0.5 \mathrm{pg}$ for TBBPA-MHEE and $0.6 \mathrm{pg}$ for TBBPA-MGE. The IDLs were also detected by HPLC-ESI-MS/MS (Quattro Premier XE, Waters, USA), and the IDLs (determined by three times of signal to noise ratio) for TBBPA-MHEE and TBBPA-MGE were $0.6 \mathrm{pg}$ and $1.0 \mathrm{pg}$, respectively. The IDLs of Orbitrap Fusion TMS were lower than that of HPLC-ESI-MS/MS. Orbitrap Fusion TMS analyzes compounds without complicated optimization of equipment parameters, and gives more accurate $\mathrm{m} / \mathrm{z}$ value for the identification of novel contaminants in environmental samples than low resolution HPLC-ESI-MS. The isotope distribution data for TBBPA-MHEE and TBBPA-MGE could be positively observed from the results of Orbitrap Fusion TMS. Orbitrap Fusion TMS showed more advantages for the detection of TBBPA-MHEE and TBBPA-MGE with highly accurate $\mathrm{m} / \mathrm{z}$ values and higher sensitivity than HPLC-MS/MS.

The method DLs (MDLs) were based on replicate analyses $(n=6)$ of blank river samples $(200 \mathrm{~mL})$ spiked at a mass concentration of $1 \mathrm{ng} / \mathrm{L}$. MDL was calculated following the Eq. (1) reported in literature $[34,35]$. MDLs of TBBPA-MHEE and TBBPA-MGE were $0.9 \mathrm{ng} / \mathrm{L}$ and $0.8 \mathrm{ng} / \mathrm{L}$. The method quantification limits (MQLs), defined as 3 times of MDLs, were 2.7 and $2.4 \mathrm{ng} / \mathrm{L}$ for TBBPA-MHEE and TBBPAMGE, respectively.

$M D L=I+3.1 * S$

$I$ is the intercept calculated from the least squared regression analysis as mentioned in linearity measurement section, $S$ is the standard deviation of the six replicate determinations of results of spiked blank samples at a mass concentration of $1 \mathrm{ng} / \mathrm{L}(0.2 \mathrm{ng}$ spiked in $200 \mathrm{~mL}$ water sample), 3.1 is the $t$-test distribution coefficient, $n=6,0.01$ level.

The recovery was determined by repeating the spike of the waste water samples $(200 \mathrm{~mL})$ at the mass concentration of $500 \mathrm{ng} / \mathrm{L}(n=5), 50 \mathrm{ng} / \mathrm{L}(n=7)$ and $5 \mathrm{ng} / \mathrm{L}(n=7)$. The recoveries at different spiking mass concentration ranged from $59 \%$ to $101 \%$ with the standard deviations (SDs) all below 14\% (Table 1). This method was also used for tap water sample to test the adaptability. When TBBPA-MHEE and TBBPA-MGE were spiked in tap water $(200 \mathrm{~mL})$ at a mass concentration of $500 \mathrm{ng} / \mathrm{L}$, the recoveries were both $85 \%$, and SDs were $4 \%$ and $6 \%$, respectively. The pretreatment method for water samples was reliable and repeatable which could satisfy the requirement of the sample analysis. The method was used to enrich the target compounds in water samples.

\subsection{Assessment of matrix effects}

In order to assess the accuracy of the method, it is necessary to evaluate potential matrix effects which can cause signal enhancement or signal suppression for the target compounds. The matrix effects of water samples were determined according to methods described in the literature $[15,36-38]$ and calculated with the following Eq. (2).

Matrix Effect $=\frac{A_{M S}-A_{M}}{A_{S}}$

$A_{\mathrm{S}}$ is the mass peak area of analyte at mass concentration of $1 \mathrm{ng} / \mathrm{mL}(0.2 \mathrm{ng} / \mathrm{mL}$ and $20 \mathrm{ng} / \mathrm{mL}$ were also tested $)$ dissolved in methanol. The waste/river water samples were extracted with DCM, re-dissolved in $1 \mathrm{~mL}$ methanol and analyzed by UHPLCOrbitrap Fusion TMS to obtain $A_{\mathrm{M}}$. Then the standards were spiked in the extract at the concentration of $1 \mathrm{ng} / \mathrm{mL}(0.2 \mathrm{ng} / \mathrm{mL}$ and $20 \mathrm{ng} / \mathrm{mL}$ were also tested) and analyzed by UHPLC-Orbitrap Fusion TMS again to obtain $A_{M S}$. If the value of the above calculation is above 1.0, there is signal enhancement, whereas if below 1.0 , there is signal suppression. If the value is 1.0 , there is no matrix effect.

The waste and river water samples were used to calculate the matrix effects. As shown in Fig. 2, no peaks relative to TBBPA-MHEE and TBBPA-MGE were detected at retention times of $3.5 \mathrm{~min}$ and $5.0 \mathrm{~min}$ both in waste water and river water samples in the spectra of matrix blank (Fig. 2, M). Although one interference peak for TBBPA-MGE was detected in waste water samples at the retention time of $4.6 \mathrm{~min}$, it would not affect the quantification of TBBPA-MGE in samples because the interference peak and TBBPA-MGE (retention time of $5.0 \mathrm{~min}$ ) could be separated by baseline (Fig. 2, MS). The peak areas of pure standards (Fig. 2, S) were comparable to those spiked in matrix blank (Fig. 2, MS).

The matrix effects were detected in three parallel experiments. The matrix effects for the waste water samples ranged from 0.93 to 1.08 , and the ones for river water samples ranged from 0.91 to 1.10 for TBBPA-MHEE and TBBPA-MGE. This indicated that the matrix effects of water samples could be ignored (Table 1 ).

\subsection{Analysis of technical products and water samples}

\subsubsection{TBBPA-MHEE and TBBPA-MGE in technical products}

The technical products were dissolved in methanol at a mass concentration of $100 \mu \mathrm{g} / \mathrm{mL}$ and analyzed with UHPLC-Orbitrap 

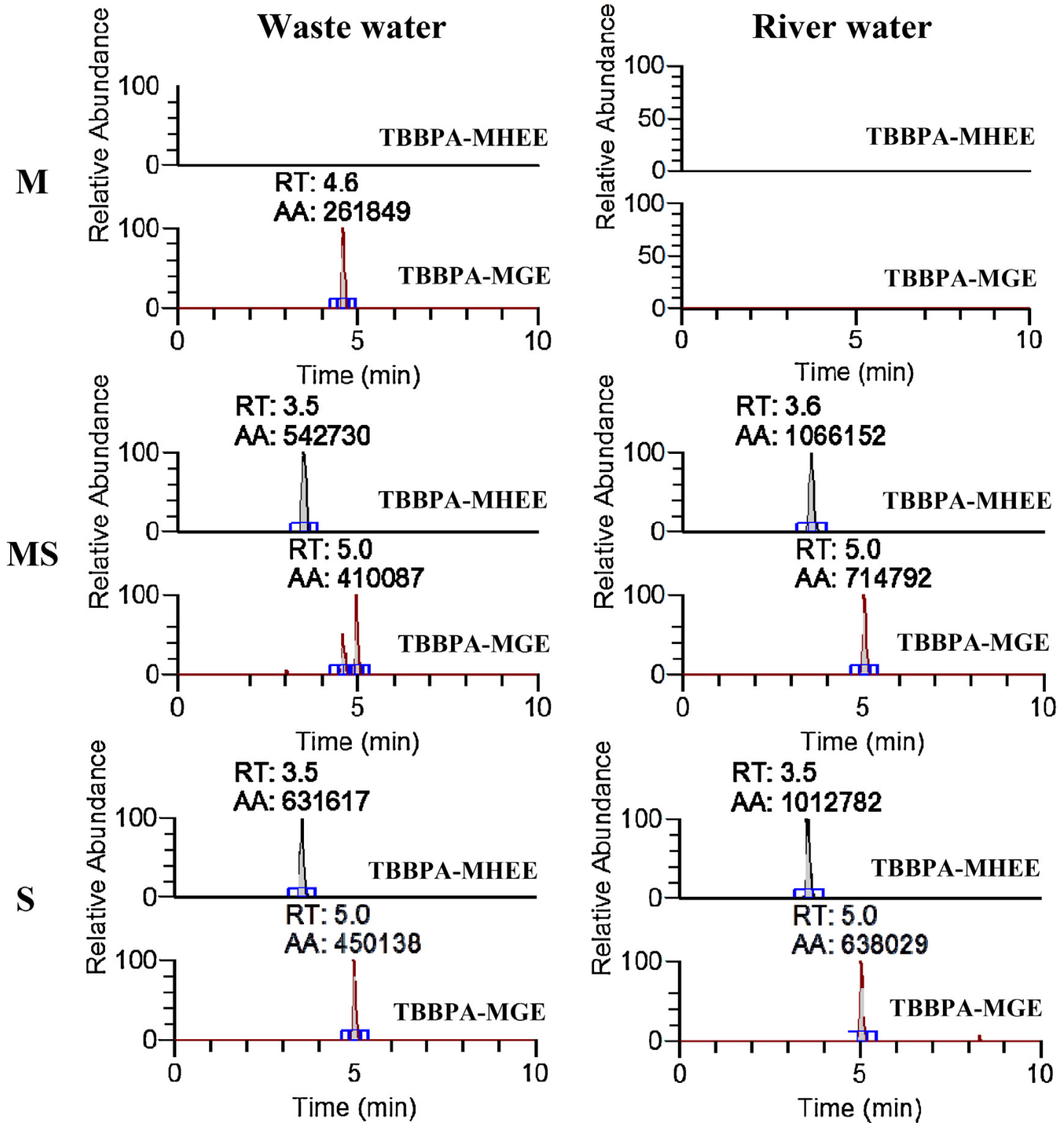

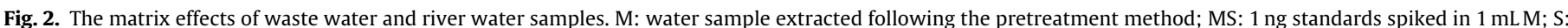
$1 \mathrm{ng}$ standards spiked in $1 \mathrm{~mL}$ methanol.

Table 2

The mass concentrations of TBBPA-MHEE and TBBPA-MGE in water samples and technical products.

\begin{tabular}{|c|c|c|c|}
\hline \multirow[t]{2}{*}{ Sample type } & & \multicolumn{2}{|c|}{$\begin{array}{l}\text { Mass concentration of detected compound } \\
\text { (water, ng/L; technical products, } \mathrm{mg} / \mathrm{kg} \text { ) }\end{array}$} \\
\hline & & TBBPA-MHEE & TBBPA-MGE \\
\hline \multirow[t]{3}{*}{ Waste water } & $\mathrm{S} 1$ & 88.5 & $<\mathrm{MDL}$ \\
\hline & S2 & 1.5 & $<\mathrm{MDL}$ \\
\hline & S3 & 5.5 & 6.5 \\
\hline \multirow[t]{3}{*}{ River water } & S4 & $<\mathrm{MDL}$ & $<\mathrm{MDL}$ \\
\hline & S5 & $<\mathrm{MDL}$ & $<\mathrm{MDL}$ \\
\hline & S6 & $<\mathrm{MDL}$ & $<\mathrm{MDL}$ \\
\hline \multirow[t]{4}{*}{ Sea water } & S7 & $<\mathrm{MDL}$ & 1.0 \\
\hline & S8 & $<\mathrm{MDL}$ & $<\mathrm{MDL}$ \\
\hline & S9 & $<\mathrm{MDL}$ & $<\mathrm{MDL}$ \\
\hline & S10 & $<\mathrm{MDL}$ & $<\mathrm{MDL}$ \\
\hline \multirow[t]{3}{*}{ Technical product TBBPA-BHEE } & Factory A & 160 & - \\
\hline & Factory B & 150 & - \\
\hline & Factory C & 160 & - \\
\hline \multirow[t]{2}{*}{ Technical product brominated epoxy resin } & Factory D & - & 46 \\
\hline & Factory E & - & 130 \\
\hline
\end{tabular}


Fusion TMS. The results showed that the mass concentration of TBBPA-MHEE in TBBPA-BHEE technical products from factory $A, B$, and $C$, were $160 \mathrm{mg} / \mathrm{kg}, 150 \mathrm{mg} / \mathrm{kg}$ and $160 \mathrm{mg} / \mathrm{kg}$, respectively (Table 2). In technical brominated epoxy resin of factory D, TBBPA-MGE was detected at a mass concentration of $46 \mathrm{mg} / \mathrm{kg}$. In technical brominated epoxy resin of factory $\mathrm{E}$, TBBPA-MGE was detected at a mass concentration of $130 \mathrm{mg} / \mathrm{kg}$ (Table 2). TBBPA-MHEE and TBBPA-MGE could be produced as impurities in the manufacturing process of technical products. Therefore, the impurities of TBBPA-BHEE, TBBPA-BGE and brominated epoxy resins of the BFR factories could be the pollution source of TBBPA-MHEE and TBBPA-MGE in environmental samples.

\subsubsection{TBBPA-MHEE and TBBPA-MGE in water samples}

By using the proposed method, TBBPA-MHEE and TBBPA-MGE presence was determined in ten water samples. TBBPA-MHEE was detected in 3 factory waste water samples at levels of 1.5-88.5 ng/L, and TBBPA-MGE was detected in one waste water sample and one sea water sample at the mass concentration of $6.5 \mathrm{ng} / \mathrm{L}$ and $1.0 \mathrm{ng} / \mathrm{L}$, respectively (Table 2). The samples near the BFR factories showed higher detection frequency and higher mass concentration of TBBPA-MHEE and TBBPA-MGE. The results indicated these new contaminants could leak into the environment through the manufacturing process of the BFR factories.

As impurities of technical products, TBBPA-MHEE and TBBPAMGE represent novel contaminants in environmental matrices with potential exposure risks to human and wildlife. Further research is needed to illuminate their distribution in environment and other pollution source, such as the degradation of TBBPA derivatives. Our results for the analyses of TBBPA-MHEE and TBBPA-MGE will further benefit the research on novel BFRs in the environment.

\section{Conclusions}

We reported for the first time the mass spectra and the analytical method for the determination of TBBPA-MHEE and TBBPA-MGE, two potential novel brominated contaminants. Meanwhile, we synthesized high purity chemicals of TBBPA-MHEE and TBBPA-MGE, which could be used for further study of their environmental behavior and toxicity. By using the high resolution UHPLC-Orbitrap Fusion TMS, details about isotope distribution in the molecular could be revealed with the quantification of TBBPA-MHEE and TBBPA-MGE at the same time. Together with qualitative information, UHPLC-Orbitrap Fusion TMS also offered higher analysis accuracy and sensitivity than HPLC-MS/MS, suggesting it is a powerful tool for the identification and quantification of novel contaminants in the environment. The pretreatment method showed high recoveries and good repeatability without matrix effects. TBBPA-MHEE and TBBPA-MGE were detected in technical products and water samples. These results indicated TBBPA-MHEE and TBBPA-MGE were mono-modified impurities synthesized with TBBPA derivatives and could leak into the environment with the production process.

\section{Acknowledgments}

This work was supported by the National Natural Science Foundation of China (No. 21377155), the Strategic Priority Research Program of the Chinese Academy of Sciences (No. XDB14010400), and the Chinese Academy of Sciences (No. YSW2013A01).

\section{Appendix A. Supplementary data}

Supplementary data associated with this article can be found, in the online version, at http://dx.doi.org/10.1016/j.chroma. 2014.12.038.

\section{References}

[1] A. Covaci, S. Harrad, M.A.E. Abdallah, N. Ali, R.J. Law, D. Herzke, C.A. de Wit, Novel brominated flame retardants: a review of their analysis, environmental fate and behaviour, Environ. Int. 37 (2011) 532-556.

[2] A. Covaci, S. Voorspoels, M.A.E. Abdallah, T. Geens, S. Harrad, R.J. Law, Analytical and environmental aspects of the flame retardant tetrabromobisphenol-A and its derivatives, J. Chromatogr. A 1216 (2009) 346-363.

[3] N. Ali, S. Harrad, E. Goosey, H. Neels, A. Covaci, Novel brominated flame retardants in Belgian and UK indoor dust: Implications for human exposure, Chemosphere 83 (2011) 1360-1365.

[4] R. Letcher, S. Chu, High-sensitivity method for determination of tetrabromobisphenol-S and tetrabromobisphenol-A derivative flame retardants in great lakes herring gull eggs by liquid chromatography-atmospheric pressure photoionization-tandem mass spectrometry, Environ. Sci. Technol. 44 (2010) 8615-8621.

[5] T. Shi, S.J. Chen, X.J. Luo, X.L. Zhang, C.M. Tang, Y. Luo, Y.J. Ma, J.P. Wu, X.Z. Peng, B.X. Mai, Occurrence of brominated flame retardants other than polybrominated diphenyl ethers in environmental and biota samples from southern China, Chemosphere 74 (2009) 910-916.

[6] J.R. Nyholm, R. Grabic, H.P.H. Arp, T. Moskeland, P.L. Andersson, Environmental occurrence of emerging and legacy brominated flame retardants near suspected sources in Norway, Sci. Total Environ. 443 (2013) 307-314.

[7] P.H. Howard, D.C.G. Muir, Identifying new persistent and bioaccumulative organics among chemicals in commerce, Environ. Sci. Technol. 44 (2010) 2277-2285.

[8] G. Qu, J. Shi, T. Wang, J. Fu, Z. Li, P. Wang, T. Ruan, G. Jiang, Identification of tetrabromobisphenol A diallyl ether as an emerging neurotoxicant in environmental samples by bioassay-directed fractionation and HPLC-APCI-MS/MS, Environ. Sci. Technol. 45 (2011) 5009-5016.

[9] E. Eljarrat, M.L. Feo, D. Barcelo, Degradation of Brominated Flame Retardants, in: E. Eljarrat, D. Barcelo (Eds.), Brominated Flame Retardants, HDb. Env. Chem., 16, Springer, 2011, pp. 187-202.

[10] P.H. Howard, D.C.G. Muir, Identifying new persistent and bioaccumulative organics among chemicals in commerce. III: Byproducts, impurities, and transformation products, Environ. Sci. Technol. 47 (2013) 5259-5266.

[11] P. Lopez, S.A. Brandsma, P.E.G. Leonards, J. De Boer, Methods for the determination of phenolic brominated flame retardants, and by-products, formulation intermediates and decomposition products of brominated flame retardants in water, J. Chromatogr. A 1216 (2009) 334-345.

[12] G. Qu, A. Liu, T. Wang, C. Zhang, J. Fu, M. Yu, J. Sun, N. Zhu, Z. Li, G. Wei, Y. Du, J. Shi, S. Liu, G. Jiang, Identification of tetrabromobisphenol A allyl ether and tetrabromobisphenol A 2,3-dibromopropyl ether in the ambient environment near a manufacturing site and in mollusks at a coastal region, Environ. Sci. Technol. 47 (2013) 4760-4767.

[13] G.J. van Esch, Tetrabromobisphenol A. \& Derivatives (Environmental Health Criteria 172), World Health Organization, Geneva, Switzerland, 1995.

[14] S.G. Chu, G.D. Haffner, R.J. Letcher, Simultaneous determination of tetrabromobisphenol A, tetrachlorobisphenol A, bisphenol A and other halogenated analogues in sediment and sludge by high performance liquid chromatography-electrospray tandem mass spectrometry, J. Chromatogr. A 1097 (2005) 25-32.

[15] R. Saint-Louis, E. Pelletier, LC-ESI-MS-MS method for the analysis of tetrabromobisphenol A in sediment and sewage sludge, Analyst 129 (2004) 724-730.

[16] K. Haraguchi, Y. Kato, K. Atobe, S. Okada, T. Endo, F. Matsubara, T. Oguma, Negative APCI-LC/MS/MS method for determination of natural persistent halogenated products in marine biota, Anal. Chem. 80 (2008) 9748-9755.

[17] M.A. Abdallah, S. Harrad, A. Covaci, Isotope dilution method for determination of polybrominated diphenyl ethers using liquid chromatography coupled to negative ionization atmospheric pressure photoionization tandem mass spectrometry: validation and application to house dust, Anal. Chem. 81 (2009) $7460-7467$.

[18] B. Johnson-Restrepo, D.H. Adams, K. Kannan, Tetrabromobisphenol A, (TBBPA) and hexabromocyclododecanes (HBCDs) in tissues of humans, dolphins, and sharks from the United States, Chemosphere 70 (2008) 1935-1944.

[19] H. Henry, H.R. Sobhi, O. Scheibner, M. Bromirski, S.B. Nimkar, B. Rochat, Comparison between a high-resolution single-stage Orbitrap and a triple quadrupole mass spectrometer for quantitative analyses of drugs, Rapid Commun. Mass Spectrom. 26 (2012) 499-509.

[20] G.T. Tomy, K. Pleskach, G. Arsenault, D. Potter, R. McCrindle, C.H. Marvin E. Sverko, S. Tittlemier, Identification of the novel cycloaliphatic brominated flame retardant 1,2-dihromo-4-(1,2-dibromoethyl)cyclo-hexane in Canadian arctic beluga (Delphinapterus leucas), Environ. Sci. Technol. 42 (2008) 543-549.

[21] A. Baesteros-Gomez, J. de Boer, P.E.G. Leonards, A novel brominated triazinebased flame retardant (TTBP-TAZ) in plastic consumer products and indoor dust, Environ. Sci. Technol. 48 (2014) 4468-4474.

[22] A. Ballesteros-Gomez, J. de Boer, P.E.G. Leonards, Novel analytical methods for flame retardants and plasticizers based on gas chromatography 
comprehensive two-dimensional gas chromatography, and direct probe coupled to atmospheric pressure chemical ionization-high resolution time-offlight-mass spectrometry, Anal. Chem. 85 (2013) 9572-9580.

[23] E.I. Hamelin, W. Bragg, R.L. Shaner, L.L. Swaim, R.C. Johnson, Comparison of high-resolution and tandem mass spectrometry for the analysis of nerve agent metabolites in urine, Rapid Commun. Mass Spectrom. 27 (2013) 16971704.

[24] N. Lopez-Gutierrez, R. Romero-Gonzalez, A. Garrido Frenich, J.L. Martinez Vidal, Identification and quantification of the main isoflavones and other phytochemicals in soy based nutraceutical products by liquid chromatographyorbitrap high resolution mass spectrometry, J. Chromatogr. A 1348 (2014) $125-136$.

[25] J. Wang, P.R. Gardinali, Identification of phase II pharmaceutical metabolites in reclaimed water using high resolution benchtop Orbitrap mass spectrometry, Chemosphere 107 (2014) 65-73.

[26] P. Herrero, F. Borrull, E. Pocurull, R.M. Marce, A quick, easy, cheap, effective rugged and safe extraction method followed by liquid chromatography(Orbitrap) high resolution mass spectrometry to determine benzotriazole, benzothiazole and benzenesulfonamide derivates in sewage sludge, J. Chromatogr. A 1339 (2014) 34-41.

[27] A. Yamamoto, H. Hisatomi, T. Ando, S. Takemine, T. Terao, T. Tojo, M. Yagi, D. Ono, H. Kawasaki, R. Arakawa, Use of high-resolution mass spectrometry to identify precursors and biodegradation products of perfluorinated and polyfluorinated compounds in end-user products, Anal. Bioanal. Chem. 406 (2014) 4745-4755.

[28] S. Song, M. Song, L. Zeng, T. Wang, R. Liu, T. Ruan, G. Jiang, Occurrence and profiles of bisphenol analogues in municipal sewage sludge in China, Environ. Pollut. 186 (2014) 14-19.

[29] H. Gallart-Ayala, E. Moyano, M.T. Galceran, Liquid chromatography/multi-stage mass spectrometry of bisphenol A and its halogenated derivatives, Rapid Commun. Mass Spectrom. 21 (2007) 4039-4048
[30] N. Fontanals, T. Barri, S. Bergstrom, J.-A. Jonsson, Determination of polybrominated diphenyl ethers at trace levels in environmental waters using hollow-fiber microporous membrane liquid-liquid extraction and gas chromatography-mass spectrometry, J. Chromatogr. A 1133 (2006) 41-48.

[31] X. Jiang, C. Basheer, J. Zhang, H.K. Lee, Dynamic hollow fiber-supported headspace liquid-phase microextraction, J. Chromatogr. A 1087 (2005) 289-294.

[32] J. Liu, J. Xie, Z. Ren, W. Zhang, Solvent extraction of phenol with cumene from wastewater, Desalin. Water Treat. 51 (2013) 3826-3831.

[33] I. Pérez-Silva, C.A. Galán-Vidal, M.T. Ramírez-Silva, J.A. Rodríguez, G.A. ÁlvarezRomero, M.E. Páez-Hernández, Phenol removal process development from synthetic wastewater solutions using a polymer inclusion membrane, Ind. Eng. Chem. Res. 52 (2013) 4919-4923.

[34] T. Ruan, Y.W. Wang, Q.H. Zhang, L. Ding, P. Wang, G.B. Ou, C. Wang. T. Wang G.B. Jiang, Trace determination of airborne polyfluorinated iodine alkanes using multisorbent thermal desorption/gas chromatography/high resolution mass spectrometry, J. Chromtogr. A 1217 (2010) 4439-4447.

[35] U.S. E.P.A., Compendium Method TO-1: Method for The Determination of Volatile Organic Compounds in Ambient Air Using Tenax ${ }^{\circledR}$ Adsorption and Gas Chromatography/Mass Spectrometry, U.S. Environmental Protection Agency, Washington, DC, 1984

[36] F. Gosetti, E. Mazzucco, D. Zampieri, M.C. Gennaro, Signal suppression/enhancement in high-performance liquid chromatography tandem mass spectrometry, J. Chromatogr. A 1217 (2010) 3929-3937.

[37] A. Salvador, C. Moretton, A. Piram, R. Faure, On-line solid-phase extraction with on-support derivatization for high-sensitivity liquid chromatography tandem mass spectrometry of estrogens in influent/effluent of wastewater treatment plants, J. Chromatogr. A 1145 (2007) 102-109.

[38] B.K. Matuszewski, M.L. Constanzer, C.M. Chavez-Eng, Strategies for the assessment of matrix effect in quantitative bioanalytical methods based on HPLC - MS/MS, Anal. Chem. 75 (2003) 3019-3030. 